\title{
La vulnérabilité des provinces espagnoles au covid-19 en fonction de leurs structures par âge et de co-résidence : implications pour le (dé) confinement
}

\author{
Albert Esteve, Iñaki Permanyer et Diederik Boertien, Centre d’Estudis Demogràfics
}

Le 11 mars 2020, l'Organisation Mondiale de la Santé a qualifiéla maladie du coronavirus (COVID-19) de pandémie mondiale. Depuis lors, le nombre de personnes infectées et les décès liés à cette maladie n'a cessé de croitre dans l'ensemble des pays du monde, et notamment en Espagne, l'un des pays les plus touchés et les plus vulnérables du fait de son niveau de vieillissement. Les pays font face à cette crise sanitaire avec des ressources et des politiques hétérogènes, mais une stratégie largement utilisée a été le confinement à domicile de la population et l'adoption de mesures de distanciation sociale. Le coronavirus peut infecter des personnes de tout âge, bien que la gravité des symptômes et la mortalité augmentent rapidement avec l'âge (OMS 2020). Par conséquent, la structure par âge de la population (le pourcentage de jeunes et de personnes âgées d'une population) et les schémas de co-résidence (lâge et le nombre des habitants d'un même ménage) sont des facteurs clefs qui conditionnent à la fois la vulnérabilité des populations au COVID-19 et l'efficacité des stratégies visant à atténuer les effets de cette épidémie. Dans ce numéro de Perspectives Demogràfiques nous analysons comment la structure démographique et la composition des ménages détermine la vulnérabilité au COVID-19 pour l'ensemble de l'Espagne et de ses provinces.

\section{DEMOGRAPHIE ET COVID-19}

D'un point de vue strictement démographique, deux facteurs déterminent la vulnérabilité des populations à une épidémie présentant les caractéristiques du COVID-19. La vulnérabilité s'entend ici comme la proportion de personnes d'une population qui pourrait mourir du virus. En toute logique, le premier facteur est la structure de la population, soit la proportion de personnes par tranche d'âge et en particulier la proportion de personnes âgées, les plus vulnérables. La réduction des infections pour ces groupes est une stratégie efficace pour réduire la mortalité. Les infections peuvent survenir à l’intérieur comme à l'extérieur du domicile. Le confinement réduit considérablement le nombre d’infections hors-domicile (infections primaires). Toutefois, les personnes infectées peuvent continuer à propager la maladie au sein de leur domicile tout en étant confinés (infections secondaires). C'est ici qu'entre en jeu le second facteur, à savoir la structure des ménages, c'est à dire les schémas de co-résidence de la population, et notamment ceux de la population âgée. Dans les ménages les plus denses, le risque de propagation au sein du foyer est plus élevé.

FIGURE 1. Vulnérabilité des provinces espagnoles au COVID-19: nombre de décès directs (gauche) et indirects (droite) pour 100 ooo habitants*
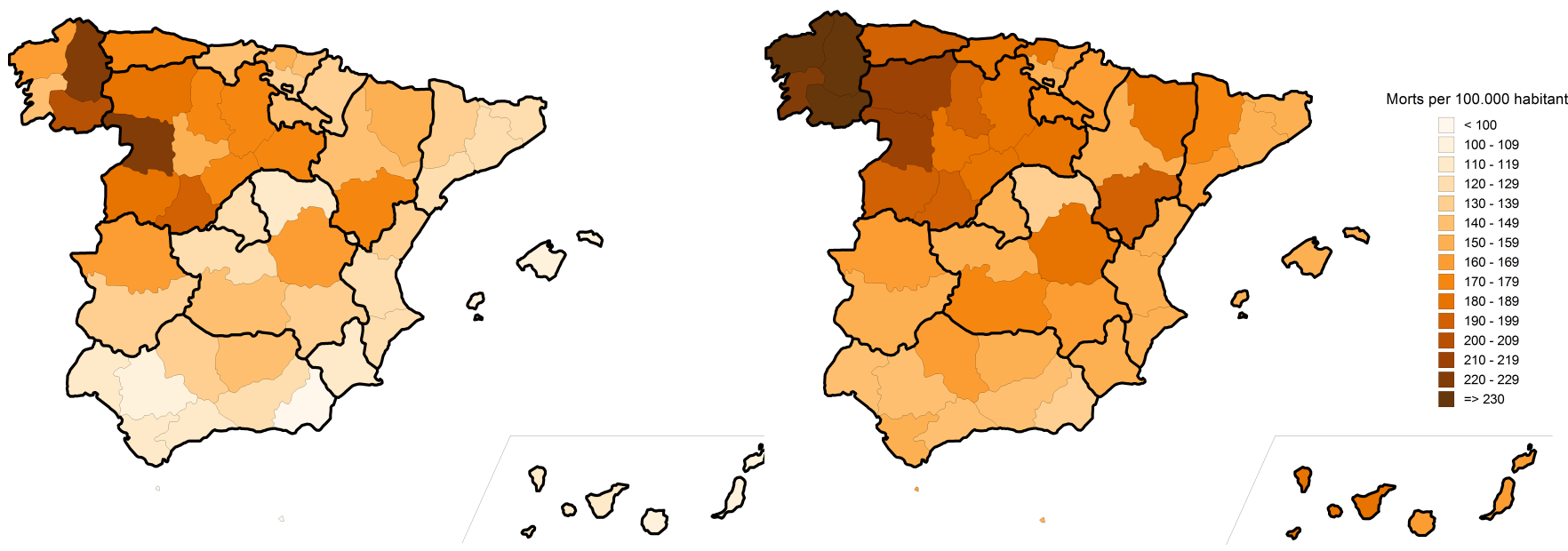

Source: Registre permanent de la population, 1/01/2018, INE-Calculs des auteurs

* Hypothèse : Infection de 10\% de la population au hasard ainsi que l'ensemble des personnes avec lesquelles elles cohabitent. 
De plus, si des personnes âgées se trouvent dans ces ménages, la mortalité se trouve à son tour plus élevée. Ces risques sont dépendants de la taille et du nombre de pièces du logement. Pour quantifier et comparer la vulnérabilité au COVID-19 des provinces espagnoles, nous avons calculé la mortalité qui aurait été observée si 10\% de la population aurait été infectée. Pour ce faire, les données du Registre de la population au 1ER janvier $2018^{1}$ ont été utilisées. Pour la simulation, l'hypothèse est faite que $10 \%$ de la population est infectée de manière aléatoire ${ }^{2}$, puis que l'infection se transmet à l'ensemble des habitants du logement d'une personne infectée ${ }^{3}$. Dans un troisième temps, les décès qui résulteraient d'infections aléatoires (décès directs) et ceux qui se produiraient à la suite d'une transmission du virus au sein du domicile (décès indirects) ont été calculés. Les décès directs seraient étroitement liés à la structure par âge de la population. Les décès indirects seraient liés à la structure du ménage, et en particulier aux logiques de cohabitation des personnes âgées.

La Figure 1 montre les taux de mortalité directe et indirecte pour 100 ooo habitants pour les différentes provinces espagnoles. La carte de gauche indique les décès directs et celle de droite les décès indirects. Les taux de mortalité directe pour 100 ooo habitants variraient entre 85 à Melilla et 216 à Zamora. Les provinces les plus vulnérables sont concentrées au nordouest du pays : Zamora (216), Lugo (213) et Orense (205). Les provinces les moins vulnérables sont situées au sud du pays : Melilla (85), Ceuta (91), Alméria (104) et Las Palmas (104). En ce qui concerne la mortalité indirecte, les taux se situeraient entre 125 à Guadalajara et 264 à Lugo. Là encore, les régions les plus vulnérables sont concentrées dans le nord-ouest du pays, les provinces galiciennes affichant les niveaux les plus élevés. A l'autre extrême de la distribution, Alméria, $65+$ $50-64$ 35-49 20-34 0-19
Granada, Huelva et Malaga seraient moins touchés par la mortalité indirecte.

Comme on peut le voir en comparant les deux cartes, la mortalité indirecte a tendance à être un peu plus élevée que la mortalité directe et ces deux mesures ne sont pas parfaitement corrélées. Cette observation donne une

Source: Registre permanent de la population, 1/01/2018, INE-Calculs des auteurs

*Hypothèse: $10 \%$ de la population infectée au hasard ainsi que l'ensemble des habitants avec lesquels ces personnes cohabitent

1 Il s'agit d'un échantillon de 10\% de la population par âge, sexe et lieu de résidence regroupé par ménage mis à disposition pour ce projet grâce à l'aimable autorisation de l'Institut National des Statistiques.

2 Les taux de mortalité par âge des personnes infectées par le COVID-19 proviennent de l'étude de Verity et al. 2020. L'utilisation de taux alternatif ou de taux de mortalité spécifiques par sexe ne fait pas varier les résultats. Ces contrôles ainsi que des contrôles supplémentaires sont disponibles sur demande auprès des auteurs. ${ }^{3}$ Il s'agit d'une hypothèse prudente qui ne doit pas nécessairement être remplie dans tous les cas mais qui démontre l'impact maximal des infections secondaires. Pour des probabilités de transmission plus faibles au sein du ménage, le nombre de décès indirects est simplement multiplié par la proportion de membres du ménage infectés de manière secondaire (par exemple, si l'on suppose que $80 \%$ des membres sont infectés, on peut multiplier le nombre de morts indirectes par o.8). 
première appréciation de l'effet multiplicateur que la structure des ménages peut avoir. En moyenne, au niveau national, seuls $45 \%$ des décès seraient attribuables aux infections primaires, le reste pouvant être attribué aux infections au sein des ménages dans le scénario où l'ensemble des habitants d'un même ménage sont infectés. Le pourcentage de décès directs sur le total des décès oscillerait significativement entre les provinces : de $37 \%$ à Melilla à 50\% à Zamora. En additionnant les taux directs et indirects, le taux de mortalité total pour 100 ooo habitants est obtenu. Ce chiffre varierait entre 231 décès à Melilla et 477 à Lugo. Il y a donc des provinces en Espagne qui, face à un niveau égal d’infections primaires, auraient jusqu'à deux fois plus de décès que d'autres régions.

La Figure 2 décompose les taux de mortalité directe et indirecte en fonction de la classe d'âge de la personne décédée, sur la base des simulations expliquées ci-dessus. Du nombre total de personnes qui décéderaient dans ce scénario, la grande majorité serait des personnes de plus de 65 ans (81\%). Les décès directs de personnes de moins de 50 ans représenteraient à peine $3 \% \mathrm{du}$ total et ceux des personnes ayant entre 50 et 64 ans environ $16 \%$. Parmi les décès indirects, plus de $73 \%$ seraient ceux de personnes de plus de 65 ans. Les moins de 50 ans représenteraient $5 \%$ du total et les 50-64 ans 22\%. Les différences entre les provinces

FIGURE 3. Répartition des âges induisant des décès indirects par contagion au sein du foyer. Espagne, Provinces*

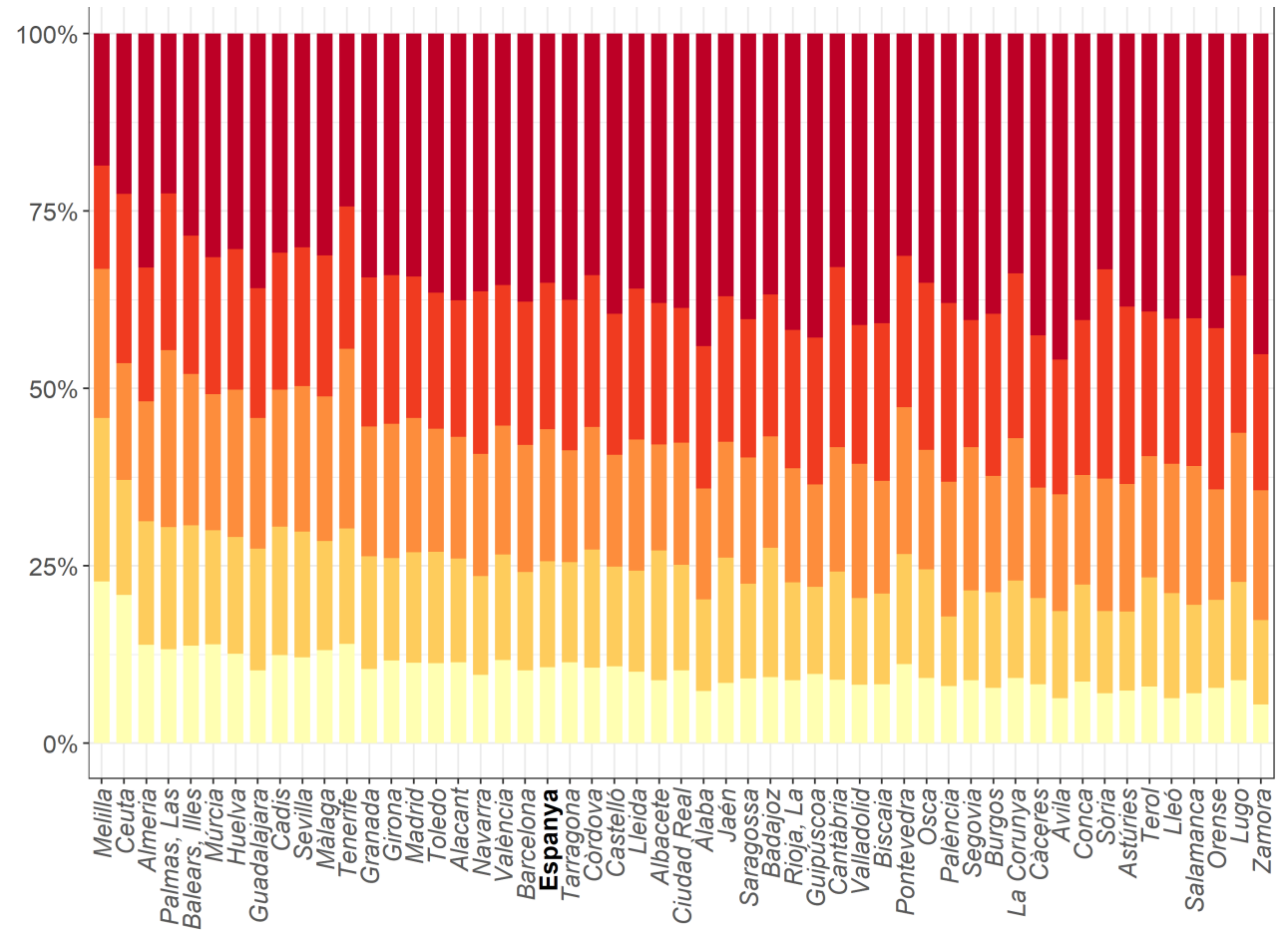

Source: Registre permanent de la population, 1/O1/2018, INE-Calculs des auteurs

${ }^{*}$ Hypothèse: $10 \%$ de la population infectée au hasard ainsi que l'ensemble des habitants avec lesquels ces personnes cohabitent. s'expliquent par (i) le pourcentage de personnes âgées de plus de 65 ans dans chaque province et (ii) les logiques de co-résidence des populations de chaque région. Le fait qu'il n'y ait pas de corrélation parfaite entre les décès directs et indirects montre que les logiques de co-résidence modulent la vulnérabilité des population de différentes manières. Dans les provinces galiciennes par exemple, les décès indirects ont des valeurs très similaires tandis que les chiffres de décès directs sont plus éloignés. Cela montre que ces provinces ont des niveaux de vieillissement légèrement différents, mais des schémas de corésidence proches.

\section{QUI CONTAMINE QUI AU SEIN DES MÉNAGES}

Dans la section précédente étaient étudiés les groupes d’âge les plus touchés par la mortalité directe et indirecte associée au COVID-19. Dans cette section, l'analyse se porte sur la distribution par âge des personnes ayant contaminé les personnes décédées des suites d'une infection au sein du domicile. En d'autres termes, est ici mesurée qui vit avec qui, en tenant compte des implications de la co-résidence sur la mortalité indirecte due aux infections au sein des ménages. L'analyse s’intéresse à l'âge des personnes infectées lors du tirage aléatoire et finissant par infecter les autres membres de leur ménage. La Figure 3 montre le pourcentage

de décès indirects Dans chaque province qui résulteraient d'infections par les autres membres du ménage en fonction de leur âge. Moins de $20 \%$ des décès indirects à Melilla 50-64 seraient provoqués par 35-49 20-34 la transmission du 0-19 virus par des personnes de plus de 65 ans et environ $20 \%$ serait le fruit d'une transmission par des enfants et des jeunes (âgés de 0 à 19 ans). En revanche, 50\% des décès indirects à Zamora seraient le fruit d'une transmission du virus par des 
personnes de plus de 65 ans alors que les enfants et les jeunes ne provoqueraient que $4 \%$ de ces décès. Dans tous les cas, la majorité des infections seraient induites par des personnes de moins de 65 ans, bien que les personnes de plus de 65 ans en soient les principales victimes, comme le montrait la Figure 2. Bien que la population jeune et en âge de travailler ait une faible probabilité de succomber des suites de la maladie, elle joue un rôle très important dans la transmission de cette dernière, lui permettant d'atteindre les populations les plus vulnérables. Les différences entre les provinces sont dues d'une part au pourcentage de personnes âgées vivant seules et d'autre part à la taille et à la composition des logements des personnes âgées cohabitant avec d'autres personnes. A Melilla par exemple, 57\% des personnes âgées de 65 ans et plus cohabitent avec des jeunes, tandis qu'à Zamora, cette proportion est de 36\%. A Zamora, 28\% des personnes âgées de 65 ans et plus vivent seules contre $36 \%$ seulement vivant avec des personnes du même groupe d'âge.

\section{UN (DÉ)CONFINEMENT EN FONCTION DE L'ÂGE?}

A l'heure d'adopter des mesures pour atténuer les effets néfastes du COVID-19, les épidémiologistes et les politiciens devraient tenir compte de la façon dont la structure par âge et les logiques de co-résidence de la population déterminent la vulnérabilité de ces dernières face à la maladie. Les études démontrent que la population âgée est la plus vulnérable et, par conséquent, les provinces où la population est plus âgée seront plus vulnérables que les provinces jeunes. Dans cette étude, ces différences ont été quantifiées et les variations inter-provinciales de la mortalité dans un scénario où 10\% de la population serait initialement infectée ont été étudiées. Parallèlement, l’importance de la transmission de la maladie au sein des ménages a été examinée.

L'une des conclusions de cette étude est que, en général, la popu- lation de moins de 65 ans pourrait provoquer plus de 50\% des décès dus à des infections au sein du domicile, bien que seulement 14\% de cette population réside avec des personnes âgées. Ce "paradoxe" s'explique par la structure démographique particulière de la population espagnole et ses schémas de co-résidence. La majeure partie de la génération du baby-boom a actuellement entre 45 et 65 ans. Bien que les niveaux de co-résidence de cette génération avec celle de ses parents ou grands-parents soient faibles en termes relatifs, le pourcentage de personnes âgées vivant avec une personne de cette génération est relativement élevé. En Espagne, 56\% des personnes de 65 ans et au-delà qui partagent un logement le font avec une personne de moins de 65 ans. De la même manière, parmi les personnes de 80 ans et plus qui ne vivent pas seules, 55\% cohabitent également avec une personne de moins de 65 ans.

Les politiques de confinement ou de "dé-confinement" devraient tenir compte de ces réalités et des différences existant entre les territoires. Les stratégies de (dé)confinement ne peuvent pas être exclusivement basées sur des critères d'âge car la population relativement jeune, et avec un risque de complications moindre, peut infecter les personnes âgées au sein des ménages. Au-delà de l'âge, les stratégies de (dé)confinement et les modèles épidémiologiques devraient intégrer plus intensivement les don- nées démographiques et de composition des ménages pour éviter, notamment, la transmission de la maladie aux personnes âgées. Des aspects comme la densité de population à l'extérieur comme à l'intérieur des logements devraient également être étudiés. Par chance, les données permettant d'analyser en profondeur la situation démographique de chaque territoire ainsi que les stratégies de confinement et de distanciation sociale les plus efficaces à mettre en place dans chaque territoires afin de minimiser le risque de contagion et l'impact du virus sur la vie quotidienne des popula- tions sont disponibles.
Références bibliographiques

Dowd, J.B. et al. (2020) "Demographic science aids in understanding the spread and fatality rates of COVID-19". medRxiv. https://doi. org/10.1101/2020.03.15.20036293

Verity, R.; Okell, L.C.; Dorigatti, I. et al (2020) "Estimates of the severity of COVID-1 disease". medRxiv preprint https://doi org/10.1101/2020.03.09.20033357

World Health Organization (2020) Coronavirus disease 2019 (COVID19): situation report, 51
Citation

Albert Esteve, Iñaki Permanyer et Diederik Boertien (2020) "La vulnérabilité des provinces espagnoles au covid-19 en fonction de leurs structures d'âge et de co-résidence : implications pour le (dé) confinement". Perspectives Demogràfiques, 19: 1-4.

Editeurs

Andreu Domingo et Albert Esteve (Centre d'Estudis Demogràfics)
Publication dirigée par:

Albert Esteve

aesteve@ced.uab.es

Crédits

Graphiques: Anna Turu et Toni Medina

Mise en page: Xavier Ruiz Vilchez

Traduction: Julien Giorgi

Adresse url

http://ced.uab.es/difusion/butlletiperspectives-demografiques
Contact

Centre d'Estudis Demogràfics. Carrer de Ca n’Altayó, Edifici E2 Universitat Autònoma de Barcelona o8193 Bellaterra / Barcelona Espanya

Email: +3493581306o Correu:demog@ced.uab.es Web: http://ced.uab.es/

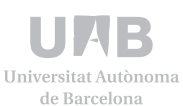

